\title{
Planetary companions around the metal-poor star HIP 11952
}

\author{
J. Setiawan ${ }^{1}$, V. Roccatagliata ${ }^{2,1}$, D. Fedele ${ }^{3}$, Th. Henning ${ }^{1}$, A. Pasquali ${ }^{4}$, M. V. Rodríguez-Ledesma ${ }^{1,5}$, E. Caffau ${ }^{4}$, \\ U. Seemann ${ }^{5,6}$, and R. J. Klement ${ }^{7,1}$
}

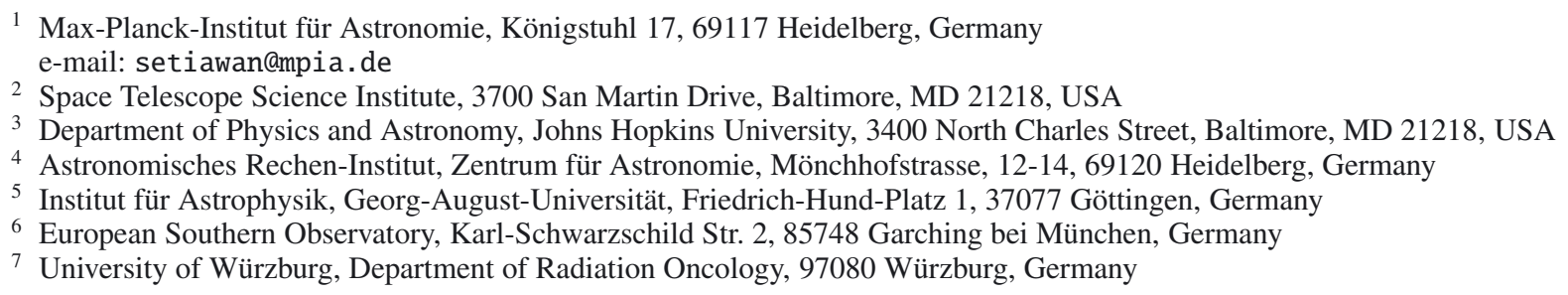

Received 4 August 2011 / Accepted 27 February 2012

\begin{abstract}
Aims. We carried out a radial-velocity survey to search for planets around metal-poor stars. In this paper we report the discovery of two planets around HIP 11952, a metal-poor star with $[\mathrm{Fe} / \mathrm{H}]=-1.9$ that belongs to our target sample.

Methods. Radial velocity variations of HIP 11952 were monitored systematically with FEROS at the $2.2 \mathrm{~m}$ telescope located at the ESO La Silla observatory from August 2009 until January 2011. We used a cross-correlation technique to measure the stellar radial velocities (RV).

Results. We detected a long-period RV variation of $290 \mathrm{~d}$ and a short-period one of $6.95 \mathrm{~d}$. The spectroscopic analysis of the stellar activity reveals a stellar rotation period of $4.8 \mathrm{~d}$. The HIPPARcos photometry data shows intra-day variabilities, which give evidence for stellar pulsations. Based on our analysis, the observed RV variations are most likely caused by the presence of unseen planetary companions. Assuming a primary mass of $0.83 M_{\odot}$, we computed minimum planetary masses of $0.78 M_{\mathrm{Jup}}$ for the inner and $2.93 M_{\mathrm{Jup}}$ for the outer planet. The semi-major axes are $a_{1}=0.07 \mathrm{AU}$ and $a_{2}=0.81 \mathrm{AU}$, respectively.

Conclusions. HIP 11952 is one of very few stars with $[\mathrm{Fe} / \mathrm{H}]<-1.0$ which have planetary companions. This discovery is important to understand planet formation around metal-poor stars.
\end{abstract}

Key words. stars: abundances - techniques: spectroscopic - planetary systems - techniques: radial velocities stars: individual: HIP 11952

\section{Introduction}

Current results of the exoplanet surveys strongly suggest a correlation between a star's stellar metallicity and its probability of hosting planets, in particular for main-sequence stars (e.g., Fischer \& Valenti 2005; Johnson et al. 2010). According to these studies, the detection rate of planets decreases with metallicity. However, the conclusions of Fischer \& Valenti (2005) might be affected by an observational bias, since these authors did not have similar numbers of stars in their survey per bin of metallicity. Therefore, it is crucial to understand if either the high stellar metallicity triggers planet formation or the metal enhancement of stars is caused by the formation of planets.

In the last years, exoplanet surveys tried to bridge this gap, starting to include more metal-poor stars in their samples. Sozzetti et al. (2009) conducted a three-year RV survey to look for planets around metal-poor stars down to $[\mathrm{Fe} / \mathrm{H}]=-2.0$ and found no evidence for short-period giant planets within $2 \mathrm{AU}$ from the central star. Santos et al. (2011) performed a similar survey, only down to $[\mathrm{Fe} / \mathrm{H}]=-1.4$ and found three moderately metal-poor stars hosting a long period giant planets $(P>700 \mathrm{~d})$. A hot Saturn and a hot Jupiter have been found transiting two moderate metal-poor stars, with $[\mathrm{Fe} / \mathrm{H}]=-0.46$ and -0.4 , respectively (Bouchy et al. 2010; Simpson et al. 2011).

In June 2009 we started a survey to search for planets around metal-poor stars. The target sample includes 96 metal-poor A and $\mathrm{F}$ stars. Our target list includes stars with metallicities in the range $-4.0 \leq[\mathrm{Fe} / \mathrm{H}] \leq-0.5$. As part of this work, Setiawan et al. (2010) found a planet around an extremely metal-poor red horizontal branch star with a short period of $16.2 \mathrm{~d}$. We notice that its $[\mathrm{Fe} / \mathrm{H}]=-2.1$ is not included in the metallicity range covered by the surveys of Sozzetti et al. (2009) and Santos et al. (2011).

These recent observations have started to disclose the realm of planets at rather low stellar metallicities, indicating that metallicity may not be the main driver of planet formation. Clearly, more statistics is needed to obtain robust conclusions. In this framework, we report the detection of two planetary companions around HIP 11952 as a result of our RV planet survey around metal-poor stars.

The paper is organized as follows: Observations and data reduction are presented in Sect. 2. The stellar parameters of HIP 11952 are shown in Sect. 3.1 together with the most relevant information available for this star in the literature. In Sect. 3.2 we describe the RV and photometric analysis. The detection of the planetary companion is addressed in Sect. 4. Discussion and conclusions are given in Sects. 5 and 6, respectively.

\section{Observations and data reduction}

We observed HIP 11952 from August 2009 until January 2011 with FEROS at the $2.2 \mathrm{~m}$ Max-Planck Gesellschaft/European 
Southern Observatory (MPG/ESO) telescope, located at ESO-La Silla observatory, during the MPG guarantee time. FEROS has a resolution of $R=48000$ and a wavelength coverage of 370-920 nm (Kaufer \& Pasquini 1998). The long-term RV precision of FEROS was measured across a period of 6 years, from December 2003 until January 2010, using the standard star $\tau$ Ceti (HD 10700). We obtained an RV precision of better than $11 \mathrm{~m} \mathrm{~s}^{-1}$.

The data reduction was performed with a software package in the ESO-MIDAS environment available online at the telescope. The procedure of the RV computation from the FEROS spectra is described in Setiawan et al. (2003) and based on a cross-correlation technique. The spectra of HIP 11952 were cross-correlated with a template of an $\mathrm{F}$ dwarf star which best matches the spectral type of HIP 11952.

\section{Analysis}

In this section we present the analysis of the stellar parameters and RVs derived from the FEROS spectra.

\subsection{The star HIP 11952}

HIP 11952 (HD 16031; LP 710-89) was previously classified as an F0 dwarf star (e.g., Wright et al. 2003; Kharchenko \& Roeser 2009) and as a giant G8 (e.g. Sánchez-Blázquez et al. 2006; Cenarro et al. 2007).

Our spectral classification was carried out by comparing the FEROS spectrum of HIP 11952 with spectra from the Indo-US library (Valdes et al. 2004) of metal-poor stars with different luminosity classes and spectral types. The FEROS spectrum was convolved to the resolution of the Indo-US spectral library ( $1 \AA$ ). Following the spectral classification criteria presented by Gray \& Corbally (2009), we used some lines (Fe II, Ti II) which are sensitive to the luminosity class of the star as well as other iron (Fe I) and calcium (CaI) lines which do not vary with the luminosity class (Fig. 1). From this comparison we concluded that HIP 11952 is an F2V star. However, this result has to be confirmed by an independent spectroscopic analysis of the stellar spectra, as we present below.

HIP 11952 is at a distance of $115.3 \mathrm{pc}$ as derived from the parallax measurements given in the HIPPARcos catalogue (Perryman 1997). Fundamental parameters of this star were determined using our high-resolution FEROS spectra. In particular, stellar abundances, effective temperature and surface gravity were computed using the synthetic spectra from the 1D ATLAS models (Kurucz 1993; Kurucz 2005) and the fit of the $\mathrm{H} \alpha$ line to the CO5BOLD 3D model atmosphere (Caffau et al. 2011). The atomic data of the iron lines are from the Large Program "First Stars" lead by R. Cayrel, optimized for metal-poor stars (see Sivarani et al. 2004). The first attempt of abundance analysis was based on 1D ATLAS model atmospheres computed using the version 9 of the code ATLAS (Kurucz 1993; Kurucz 2005) running under Linux (Sbordone et al. 2004; Sbordone 2005). We derived a temperature of $5960 \mathrm{~K}$ by fitting the $\mathrm{H} \alpha$ wings with a grid of synthetic spectra computed from ATLAS models, and a temperature of $6120 \mathrm{~K}$ when we used a grid of synthetic spectra based on 3D models.

By imposing an agreement between the iron abundance derived from the lines of $\mathrm{Fe}_{\mathrm{I}}$ and the lines of $\mathrm{Fe}$ II, we derived surface gravities $\log g$ of 3.8 and 4.0 for the two cases of $T_{\text {eff }}=5960 \mathrm{~K}$ and $6120 \mathrm{~K}$, respectively. The uncertainty on the effective temperature derived from the fit of the $\mathrm{H} \alpha$ is $150 \mathrm{~K}$,

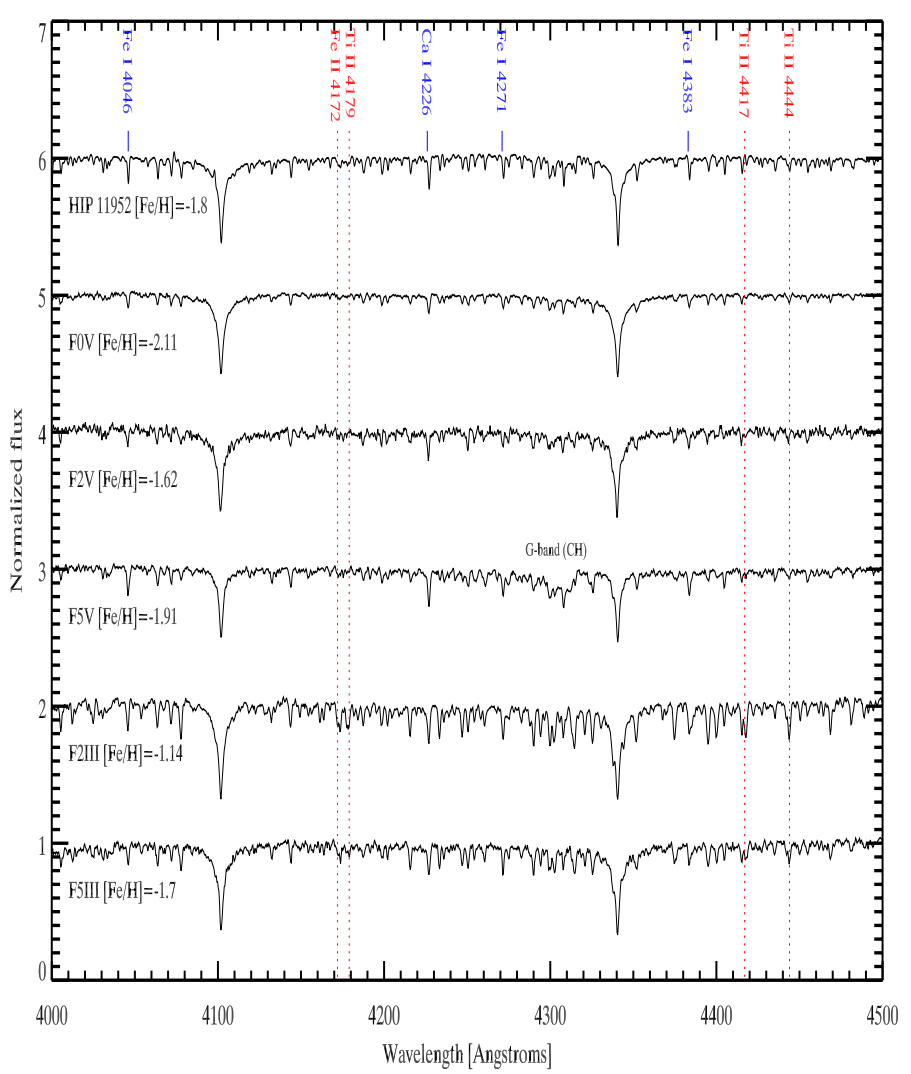

Fig. 1. Comparison of the spectrum of HIP 11952 with stellar spectra of the Indo-US library. The dashed lines highlight some iron lines (Fe II, Ti II) which are sensitive to the luminosity class of the star. The solid lines in the upper part of the figure indicate the position of some iron and calcium lines which do not vary with the luminosity class.

while the error in the surface gravity is 0.3 . A microturbulence of $1.4 \mathrm{~km} \mathrm{~s}^{-1}$ was derived by minimizing the slope of the abundance versus equivalent width $(E W)$ relation. The resulting iron abundance derived is $[\mathrm{Fe} / \mathrm{H}]=-1.95 \pm 0.09$ for $T_{\text {eff }}=5960 \mathrm{~K}$ and $[\mathrm{Fe} / \mathrm{H}]=-1.85 \pm 0.09$ for $T_{\mathrm{eff}}=6120 \mathrm{~K}$, respectively.

We thus obtained two possible parameter sets for this star: $\left(T_{\mathrm{eff}}, \log g,[\mathrm{Fe} / \mathrm{H}]\right)=(5960 \mathrm{~K}, 3.8,-1.95)(1 \mathrm{D}$ ATLAS $)$ and $(6120 \mathrm{~K}, 4.0,-1.85)$ (3D MODELS). We measured an $E W=$ $3.07 \pm 0.03 \mathrm{~mA}$ of the Li feature at $670.7 \mathrm{~nm}$, which implies an abundance $A(\mathrm{Li})=2.2$ if we fix $T_{\text {eff }}=6120 \mathrm{~K}$, and $A(\mathrm{Li})=2.1 \mathrm{in}$ the case $T_{\text {eff }}=5960 \mathrm{~K}$.

Feltzing et al. (2001) derived a stellar age of $12.8 \pm 2.6 \mathrm{Gyr}$ by comparing the Strömgren photometry of HIP 11952 with the evolutionary tracks computed for the Strömgren metallicity $([m / \mathrm{H}]=-1.54)$ of the star. We checked their result using the photometry provided by HIPPARcos for HIP 11952 in the Bessell filters system (Bessell 2000) with the isochrones by Marigo et al. (2008) and Girardi et al. (2010), calculated for the Strömgren metallicity of the star. In the assumption that the dust extinction along the line of sight to HIP 11952 is not large, the HipParcos photometry indicates, within its uncertainty, an age older than 10 Gyr for this star.

We used the isochrones by Marigo et al. (2008) and Girardi et al. (2010) computed for $[\mathrm{m} / \mathrm{H}]=-1.54$ and ages in the range 10-13 Gyr in order to consistenly derive the mass and radius of HIP 11952. By comparing its photometry with the selected isochrones, we constrained its mass between $0.79 M_{\odot}$ and 
$0.88 M_{\odot}$. From the relation between surface gravity, stellar mass and stellar radius:

$$
\frac{R}{R_{\odot}}=\sqrt{\frac{M}{M_{\odot}} \frac{g_{\odot}}{g}}
$$

we derived a stellar radius of $1.6 \pm 0.1 R_{\odot}$, where the error is obtained from the errors propagation. The derived stellar mass and radius adopted in this work are: $0.83_{-0.04}^{+0.05} M_{\odot}$ and $1.6 \pm 0.1 R_{\odot}$.

Fundamental stellar parameters have also been estimated in previous studies (e.g., Masana et al. 2006; Charbonnel et al. 2005). The corresponding values are $T_{\text {eff }}=6367 \mathrm{~K}$, a stellar radius $R_{*}=1.0 R_{\odot}$ and surface gravity $\log g=4.1$. Feltzing et al. (2001) reported $m=0.785 M_{\odot}$. If we compile all literature values for the surface gravity given in Cayrel de Strobel et al. (2001) and use $R_{*}=1.0 R_{\odot}$, we obtain a mean value $m=0.55 \pm 0.23 M_{\odot}$. Within the errors, these values are consistent with those we derived, although the stellar radius is smaller than the one we adopted. Finally, we compared the derived stellar parameters with those given by Casagrande et al. (2010). These authors measured $\log g=4.17,[\mathrm{Fe} / \mathrm{H}]=-1.74$ and $T_{\text {eff }}=6186 \mathrm{~K}$. Within the uncertainties, these values are in good agreement with our determination.

Based on the surface gravity and radius derived from our analysis and the available literature data, HIP 11952 is more likely a star already evolved off the main-sequence, roughly sitting at the base of the subgiant branch.

Astrometric and photometric data of HIP 11952 can be found in public catalogues (e.g., Hipparcos). The astrometric variability is less than 5 mas, allowing the conclusion that an unseen stellar companion in the system can be ruled out. Nevertheless, older RV measurements from Eggen \& Sandage (1959) combined with Carney \& Latham (1987) suggested that HIP 11952 may be a spectroscopic binary SB1 (Fouts 1987). However, our measurements can neither reject nor confirm this claim yet. The updated parameters of HIP 11952 are given in Table 1.

HIP 11952 is listed as a member of the metal-poor stellar stream detected by Arifyanto \& Fuchs (2006), a group of putative thick disk stars moving on similar orbits in the Galactic potential and currently lagging the local standard of rest by $V_{\text {lag }} \approx 80 \mathrm{~km} \mathrm{~s}^{-1}$. As such, HIP 11952 might stem from one of the Milky Way's former satellite galaxies that once got tidally disrupted. Alternatively, Minchev et al. (2009) showed that the stream might consist of in situ disk stars being scattered towards common orbits through a merger-induced perturbation of the old disk $\sim 1.9$ Gyr ago. It is also possible that there is a connection to the Arcturus stream at $V_{\text {lag }} \approx 100$ which itself could have a tidal or a dynamical origin (see discussion in Klement 2010, for more details).

\subsection{Radial velocity}

The RV variation of HIP 11952 is shown in Fig. 2 (upper panel). During the observation campaigns we obtained 77 RV measurements (Table 2). We applied the generalized Lomb-Scargle (GLS) periodogram (Zechmeister \& Kürster 2009) to the RV data in order to search for periodicities.

We found several signals in the periodogram, as shown in the lower panel of Fig. 2. The four highest signals are marked with $\mathrm{P}_{1}, \mathrm{P}_{2}, \mathrm{P}_{3}$ and $\mathrm{P}_{4}$. The highest peak $\mathrm{P}_{1}$ has a false alarm probability (FAP) of $7.2 \times 10^{-6}$ and corresponds to a period of $290 \pm 16$ d. We computed a Keplerian fit to the data, shown as
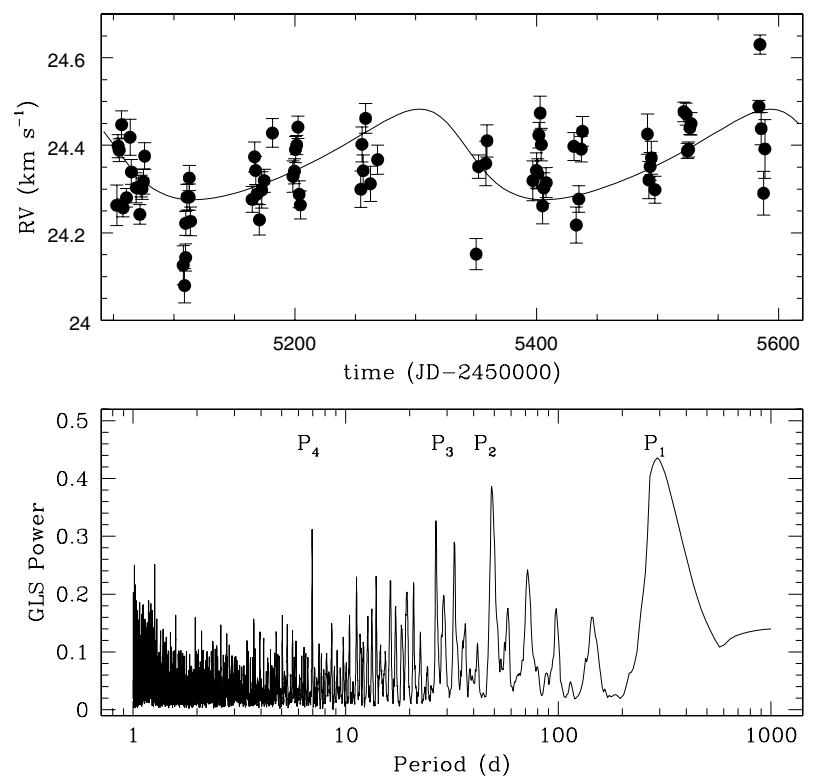

Fig. 2. RV observations of HIP 11952, taken with FEROS from August 2009 until January 2011 (upper panel). We calculated a single Keplerian fit to the data (solid line) for the outer component. A multicomponent Keplerian fit is presented in Fig. 9, see Table 3. The lower panel shows a Generalized Lomb-Scargle (GLS) periodogram of the whole RV data set. The highest peak, marked with $\mathrm{P}_{1}$ corresponds to a period of $290 \pm 16 \mathrm{~d}$ with a false alarm probability of $\sim 7 \times 10^{-6}$.

Table 1. Stellar parameters of HIP 11952.

\begin{tabular}{lcc}
\hline \hline Parameter & Value & Unit \\
\hline Spectral type $^{*}$ & F2V-IV & \\
$m_{V}{ }^{a}$ & $9.88 \pm 0.02$ & $\mathrm{mag}$ \\
Parallax $^{a}$ & $8.67 \pm 1.81$ & $\mathrm{mas}$ \\
$T_{\text {eff }}{ }^{*}$ & $5960 \pm 150$ & $\mathrm{~K}$ \\
& $6120 \pm 150$ & $\mathrm{~K}$ \\
$\log \mathrm{g}^{*}$ & $3.8 \pm 0.3$ & $\mathrm{~cm}^{2} / \mathrm{g}$ \\
& $4.0 \pm 0.3$ & $\mathrm{~cm}^{2} / \mathrm{g}$ \\
{$[\mathrm{Fe} / \mathrm{H}]^{*}$} & $-1.95 \pm 0.09$ & $\mathrm{dex}$ \\
& $-1.85 \pm 0.09$ & $\mathrm{dex}$ \\
$R_{*}{ }^{*}$ & $1.6 \pm 0.1$ & $R_{\odot}$ \\
Mass $^{*}$ & $0.83_{-0.04}^{+0.05}$ & $M_{\odot}$ \\
Age $^{b}$ & $12.8 \pm 2.6$ & $\mathrm{Gyr}$ \\
$v_{\text {rot }} \sin i^{*}$ & $5.2 \pm 1.0$ & $\mathrm{~km} \mathrm{~s}^{-1}$ \\
$P_{\text {rot }} / \sin i$ & $15.7 \pm 2.5$ & $\mathrm{~d}$ \\
\hline
\end{tabular}

Notes. ${ }^{(*)}$ This work. (a) HIPPARcos catalogue (Perryman 1997). (b) Feltzing et al. (2001).

the solid line in the upper panel of Fig. 2. The parameters of this fit are given in Table 3.

After removing the $290 \mathrm{~d}$ signal, the peaks $\mathrm{P}_{2}$ and $\mathrm{P}_{3}$ disappear. However, the peak $\mathrm{P}_{4}$ remains, which means that this signal is not an alias of $P_{1}$. The signal $\mathrm{P}_{4}$ corresponds to a period of $6.95 \mathrm{~d}$ with FAP $=8 \times 10^{-4}$. In the residual RV periodogram, we also observed a signal at $1.16 \mathrm{~d}$ (Fig. 3 upper panel), which is obviously a harmonic of the $6.95 \mathrm{~d}$ period $(1 / 6.95+1 / 1.16=$ 1.0). In the lower panel of Fig. 3 we have phase folded the RVs with $P=6.95 \mathrm{~d}$ and show a Keplerian fit with the parameters listed in Table 3. After excluding stellar activity as the cause for the RV variations in the next sections, we are going to interpret 

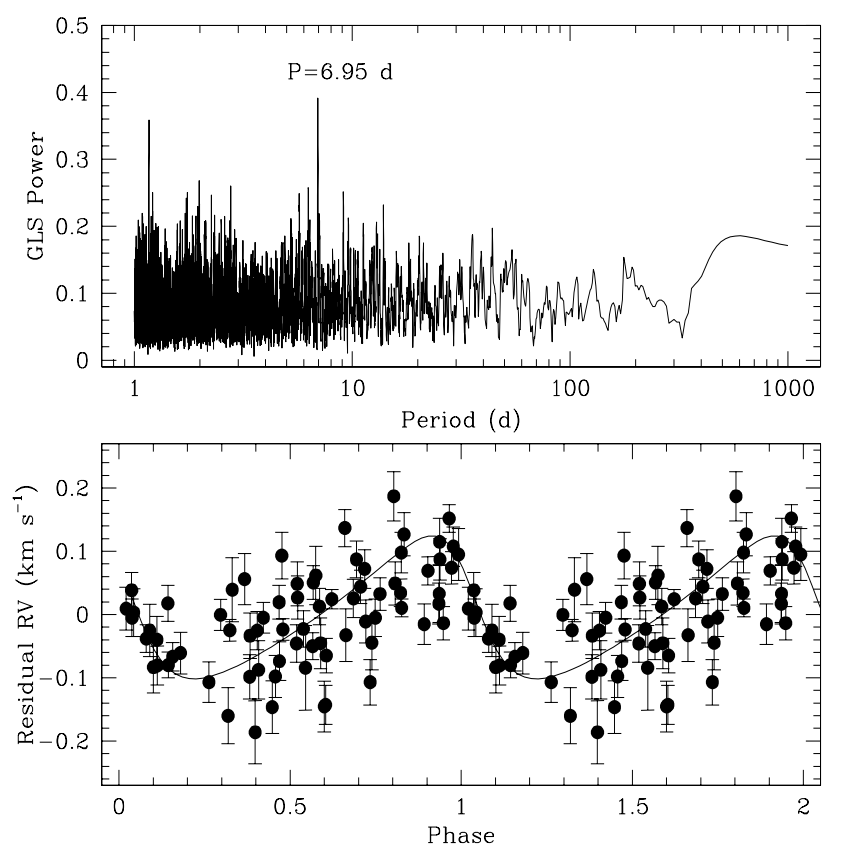

Fig. 3. The residual RV after removing the 290 d periodicity. The periodogram in the upper panel shows two peaks at $6.95 \mathrm{~d}$ and $1.16 \mathrm{~d}$. The $1.16 \mathrm{~d}$ is identified as a harmonic of $6.95 \mathrm{~d}(1 / 1.16+1 / 6.95=1.0)$. The lower panel shows the phase folded RVs with a period of $6.95 \mathrm{~d}$. The solid line shows a Keplerian fit for the residual RV variation.

the signals $P_{1}$ and $P_{4}$ as orbital periods of unseen low-mass companions (Sect. 4 below).

\subsection{Stellar rotation}

A systematic investigation of the stellar activity is mandatory to avoid wrong interpretations of the observed RV variations. There are several possibilities to probe the stellar activity. The line profile asymmetry (bisector) and the Ca II lines are known as reliable stellar activity indicators. These activity indicators, if they show periodic variations, can be used to determine the stellar rotation period. Besides the spectroscopic methods, photometric observations are also commonly used to find the stellar rotation period.

HIP 11952 itself is not a star with high stellar activity. This is inferred from the absence of emission cores in Ca II K ( $\lambda 3934)$ and $\mathrm{H}$ ( $\lambda 3967)$. Furthermore, no $\mathrm{H} \alpha$ emission line was observed in the spectra. The projected rotational velocity is also relatively low $\left(v \sin i=5.2 \mathrm{~km} \mathrm{~s}^{-1}\right)$ compared to other F-type dwarf stars (de Medeiros \& Mayor 1999). Finally, using the relation $P / \sin i=2 \pi R_{*} / v \sin i$, the maximum stellar rotation period is found to be $15.7 \pm 2.5 \mathrm{~d}$.

\subsubsection{Line profile asymmetry}

The line profile asymmetry can be quantified by the bisector velocity span (BVS). A definition of the BVS is given, e.g., in Hatzes (1996). We measured the BVS of the stellar spectra and searched for any periodicity that might be related to the RV variation. In the GLS periodogram of BVS we found, however, no significant peak. Thus, we cannot use the bisector to determine the stellar rotation period. We then searched for a correlation between BVS and RV to find out whether the observed RV variation is due to rotational modulation. We computed a correlation coefficient $c=0.1$ between the RV and BVS (Fig. 4). This
Table 2. RV variation of HIP 11952.

\begin{tabular}{|c|c|c|c|c|c|}
\hline $\begin{array}{l}\text { JD } \\
-2450000\end{array}$ & $\begin{array}{l}\mathrm{RV} \\
(\mathrm{m} / \mathrm{s})\end{array}$ & $\begin{array}{l}\text { Error } \\
(\mathrm{m} / \mathrm{s})\end{array}$ & $\begin{array}{c}\text { JD } \\
-2450000\end{array}$ & $\begin{array}{l}\mathrm{RV} \\
(\mathrm{m} / \mathrm{s})\end{array}$ & $(\mathrm{m} / \mathrm{s})$ \\
\hline 5052.8320 & 24262.99 & 46.17 & 5256.5331 & 4342.01 & \\
\hline & & & & & \\
\hline 505 & 9 & & & & \\
\hline & & & & & \\
\hline & 3 & 20 & 53 & & 5.2 \\
\hline & 280 & 21 & 88 & & $26.7 \xi$ \\
\hline & & & 51 & & 0.7 \\
\hline & & & & & 36.79 \\
\hline & & & & & 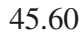 \\
\hline & & & & & .4 \\
\hline & & & & & 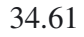 \\
\hline & & & & & \\
\hline & & & & & 0.14 \\
\hline & & & & & 7.24 \\
\hline & & & & & 1.39 \\
\hline & & & & & 5.31 \\
\hline & & & & & 4.22 \\
\hline & & & & & 32.08 \\
\hline & & 2 & & & 40.97 \\
\hline & & & & & 30.19 \\
\hline & & & & & 9.11 \\
\hline & & & & & 3 \\
\hline & & & & & $.26-2>$ \\
\hline & & & & & 42.54 \\
\hline & & & & & 04 \\
\hline & & & & & 64 \\
\hline & & & & & 3( \\
\hline & & & & & \\
\hline & & & & & \\
\hline & & & & & \\
\hline & & & & & 25 \\
\hline & & & & & 15.05 \\
\hline & 9 & & & & 25.68 \\
\hline & & & & & 14.01 \\
\hline & & & & & 2168 \\
\hline & & & & & \\
\hline & & & & & \\
\hline & & & 8.6275 & 24391.65 & 00.9 \\
\hline & & & & & \\
\hline
\end{tabular}

value indicates that the RV variation is not correlated with the BVS. However, it does not give any hint about the stellar rotation period.

\subsubsection{Ca II analysis}

As mentioned before we found no emission cores in the $\mathrm{Ca}$ II $\mathrm{H}$ \& K lines $(\lambda \lambda 3934,3968)$ which are, in general, excellent stellar activity indicators to probe the rotational modulation of the star. Nevertheless, we investigated to possibility to use Ca II K lines to find any indication of stellar activity. We calculated the stellar activity index, known as $S$-index, similar to the method described in Vaughan et al. (1978) and Santos et al. (2000). We found a significant periodicity of $36 \mathrm{~d}$ in the periodogram, but the error bar of the individual measurement is large. This value is also close to the typical observational window of about one month. Thus, also by considering $P / \sin i$ value, we do not adopt this as the stellar rotation period.

We exploited the capability of FEROS to investigate the Ca II lines $\lambda \lambda 8498,8662$, following the technique presented e.g. by Larson (1993), where Ca II $\lambda 8662$ was used to determine the stellar rotation period. Setiawan et al. (2010), for example, used the equivalent width (EW) variations of Ca II $\lambda 8498$ as a stellar 


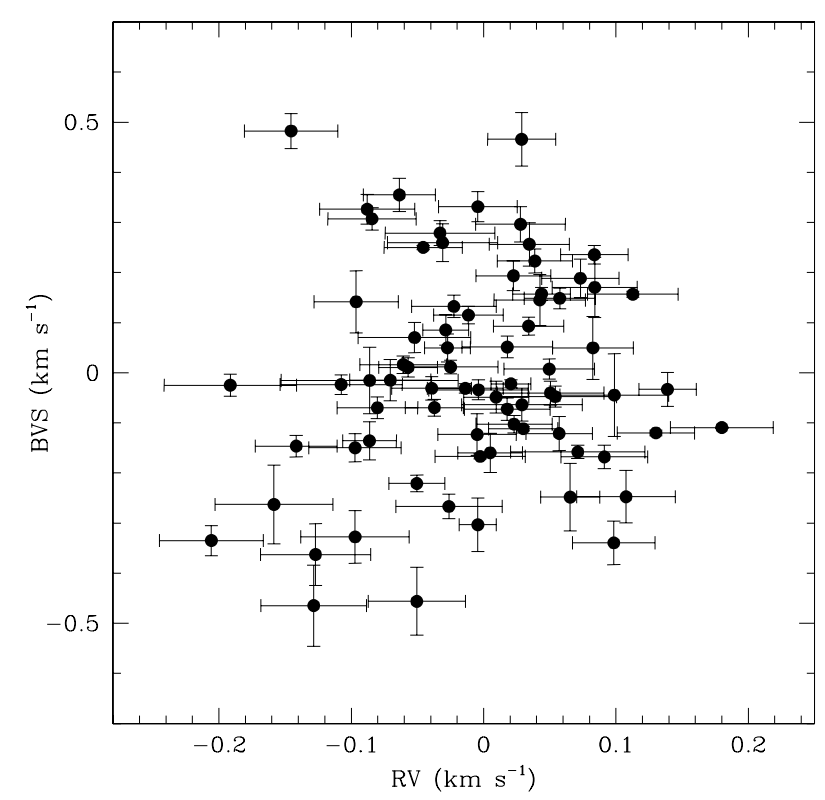

Fig. 4. The figure shows the measurements of BVS, ploted against residual RVs. The plot shows no correlation between BVS and RV. We computed a correlation coefficient $c=0.1$.
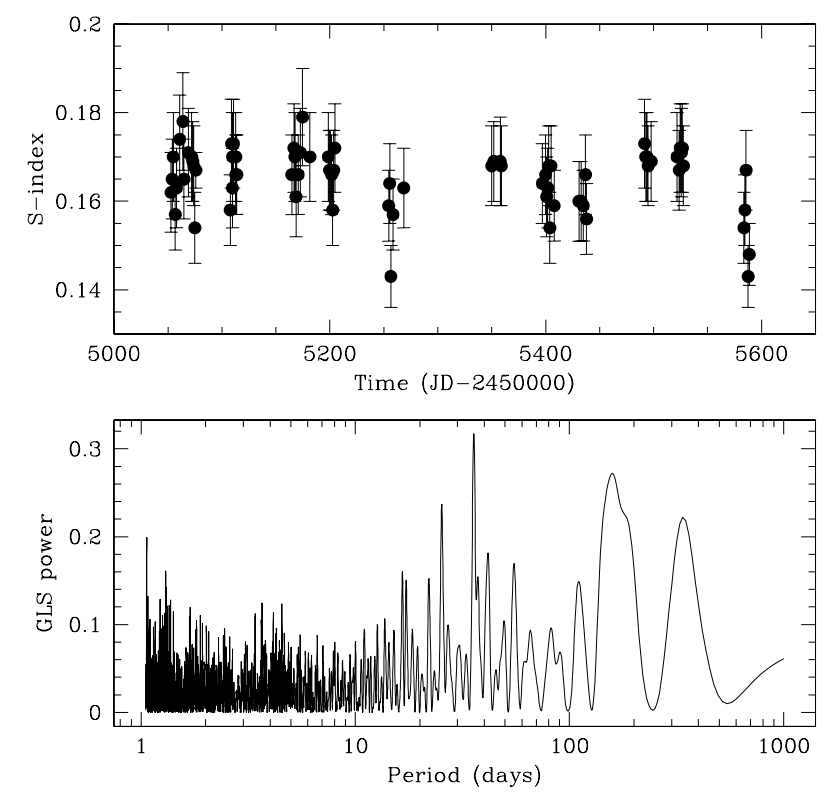

Fig. 5. Upper panel: the measurements of Ca II $\lambda 3934 S$-index. Lower panel: the GLS periodogram of Ca II $\lambda 3934$ shows a clear peak corresponding to a period of $P=36 \mathrm{~d}$. However, the error bar of the individual measurement is large and the period is close to the observational time window of about 1 month.

activity indicator to estimate a stellar rotation period that agrees with the bisector analysis.

The EW variation of Ca 18498 of HIP 11952 indeed shows a periodic variation with $P=1.76 \mathrm{~d}$ with a FAP of few percent. Thus, it is only marginaly significant. Following Larson (1993) we then measured the EW variation of Ca II $\lambda 8662$. Interestingly, we found a significant periodicity in the EW variation of Ca II 18662 . The signal corresponds to a peak at a period of $P=4.82 \mathrm{~d}$ with $\mathrm{FAP}=8 \times 10^{-3}$. Figure 6 shows
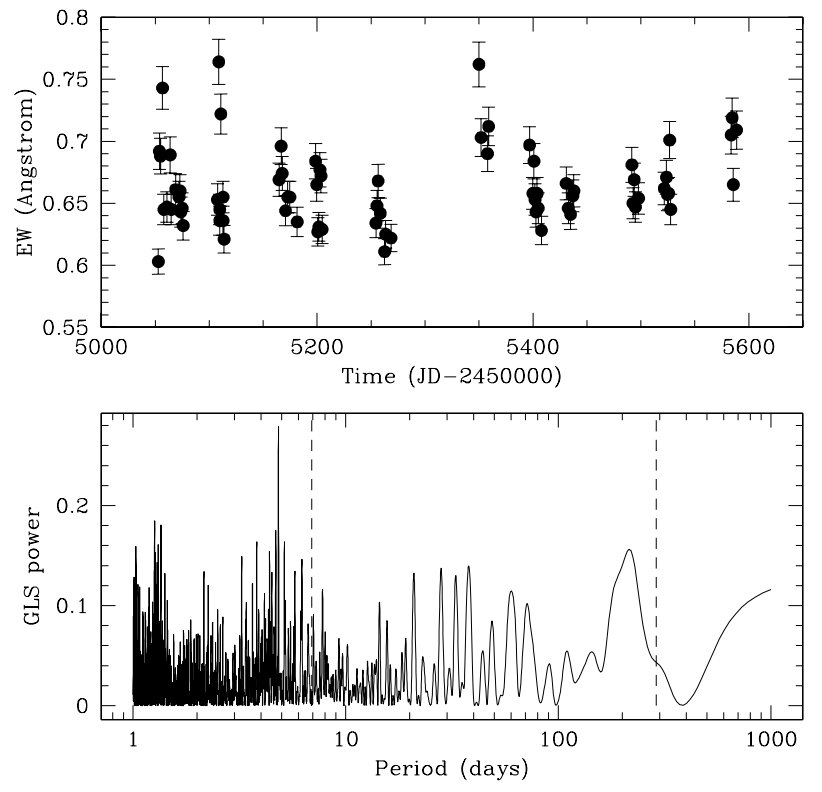

Fig. 6. Upper panel: the measurements of Ca II $18662 \mathrm{EW}$ variation. Lower panel: the GLS periodogram of Ca II 18662 shows a clear peak corresponding to a period of $P=4.82 \mathrm{~d}$. The dashed line shows the period of the RV variations.

the EW variation and GLS periodogram of the Ca II $\lambda 8662$ line. Assuming that this feature is related to the stellar magnetic activity caused by starspots, the period is most-likely linked to the stellar rotation.

\subsubsection{Photometric data}

Photometric $V$ band observations of HIP 11952 are available in the Hipparcos catalogue. Unfortunately, the data set is very sparse, with 72 photometric measurements over a time span of $893 \mathrm{~d}$. The minimum time-interval between data points is $\approx 0.014 \mathrm{~d}$, and the typical photometric errors are $\approx 0.02 \mathrm{mag}$. Due to long-term gaps of several days in the data set, periodicities of a few days cannot be reliably detected. The sampling allows for the detection of very short-term (few hours) as well as long-term (20 d) variations.

We searched for periodicity in the photometric data using a combination of two periodogram analysis techniques: the Scargle periodogram (Scargle 1982) and the CLEAN algorithm (Roberts 1987). The combination of these two techniques provides a reliable period detection as outlined in several rotational period and variability studies (e.g., Rodríguez-Ledesma et al. 2009).

Based on the Scargle periodogram, we detected significant signals at $P_{1}=0.072, P_{2}=0.33$ and at $P_{3}=2 \mathrm{~d}$. When the CLEAN algorithm is applied, the $2 \mathrm{~d}$ signal is removed and therefore, we concluded that it is probably a false peak or alias due to the clumpy data sampling. Both the 0.072 and $0.33 \mathrm{~d}$ periods in the power spectrum remains (Fig. 7), with FAP, based on the Scargle periodogram, of $0.5 \%$ and $1 \%$, respectively. All other peaks in Fig. 6 have larger FAPs. We have also computed the statistical F-test and a derived FAP from it (Scholz et al. 2004; Rodríguez-Ledesma et al. 2009). The FAP Ftest $_{\text {represents }}$ the probability that the period found is caused by variations in the photometric noise, and therefore it is independent of the periodogram analysis. The FAP Ftest derived for the 0.072 and 


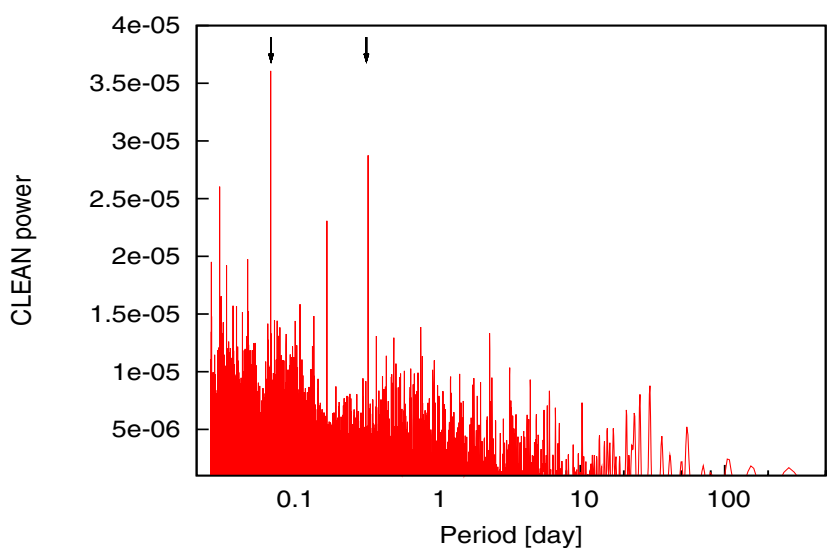

Fig. 7. The photometric observations of HIP 11952 show two peaks at $P=0.3$ and $P=0.072 \mathrm{~d}$.
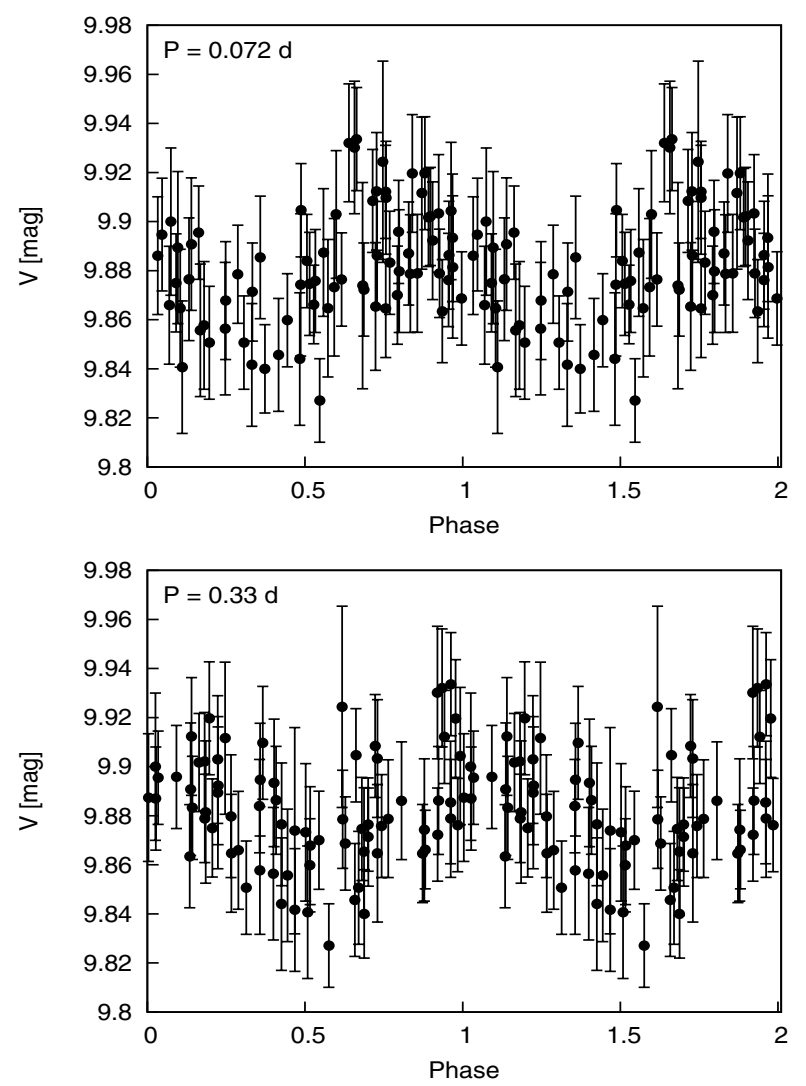

Fig. 8. Phase folded light curves from the HiPparcos photometric data.

$0.33 \mathrm{~d}$ detected peaks are $5 \%$ and $11 \%$, respectively. Figure 8 shows the phase folded light curves with the periods of 0.072 and $0.33 \mathrm{~d}$.

Due to the quality of the data set, however, it is difficult to ensure the significance of these periods. Our analysis suggests some evidence for short-term photometric variations, which might be interpreted as possible pulsation modes in this F-type star.

Based on the analysis of the stellar activity indicators, we assume a stellar rotation period of $4.8 \mathrm{~d}$, as derived from the Ca II 18662 . Additionally, there might be evidence for stellar pulsations with intra-day periodicities

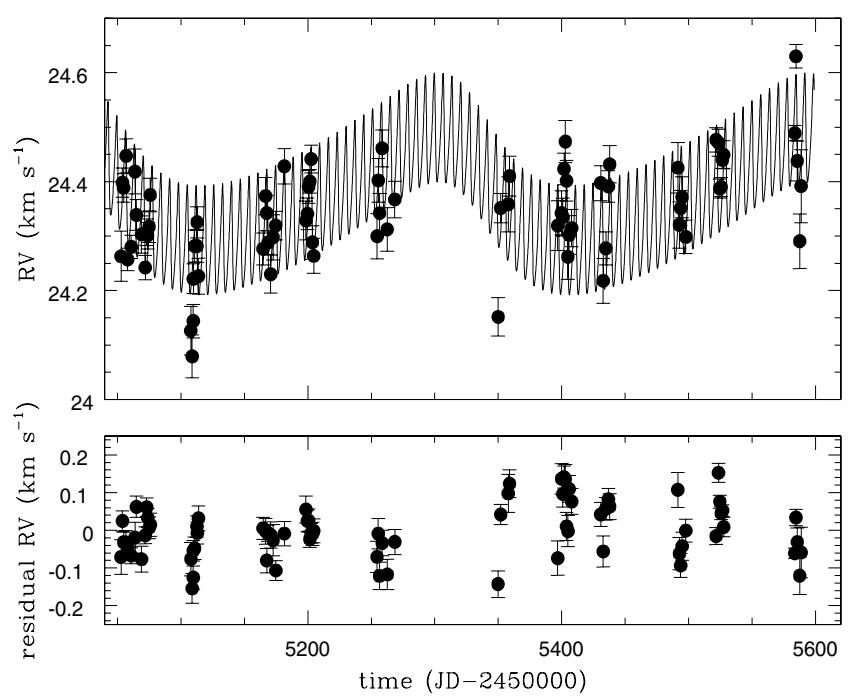

Fig. 9. Orbital solution of a two-planets Keplerian fit (upper panel). The orbital parameters are listed in Table 3. The residual velocities are shown in the lower panel.

Table 3. Orbital parameters for HIP $11952 \mathrm{~b}$ and c.

\begin{tabular}{lccc}
\hline \hline Parameter & Unit & HIP 11952b & HIP 11952c \\
\hline$P$ & $\mathrm{~d}$ & $290.0 \pm 16.2$ & $6.95 \pm 0.01$ \\
$T_{0}$ & $\mathrm{JD}$ & $5402.0 \pm 1.3$ & $5029.2 \pm 0.04$ \\
& -2450000 & & \\
$e$ & & $0.27 \pm 0.10$ & $0.35 \pm 0.24$ \\
$\omega_{1}$ & $\mathrm{deg}$ & $59.3 \pm 2.5$ & $61.2 \pm 6.6$ \\
$K_{1}$ & $\mathrm{~m} \mathrm{~s}^{-1}$ & $105.2 \pm 14.7$ & $100.3 \pm 19.4$ \\
$m_{2} \sin i$ & $M_{\text {Jup }}$ & $2.93 \pm 0.42$ & $0.78 \pm 0.16$ \\
$a$ & $\mathrm{AU}$ & $0.81 \pm 0.02$ & $0.07 \pm 0.01$ \\
\hline$V_{0}$ & $\mathrm{~km} \mathrm{~s}^{-1}$ & $24.365 \pm 0.01$ \\
$\sigma(\mathrm{O}-\mathrm{C})$ & $\mathrm{m} \mathrm{s}^{-1}$ & 70.22 \\
reduced $\chi^{2}$ & \multicolumn{3}{|}{1.3} \\
\hline
\end{tabular}

\section{Planetary companion}

Since the long-period and short-period RV variations have different characteristics from those of the stellar activity indicators, we concluded that they are most likely caused by the presence of unseen companions.

We computed the orbital solution by using a twocomponents Keplerian model. In Fig. 9 we show the calculated orbital fit and residual velocities. The orbital paramaters are given in Table 3 . With a derived primary mass of $0.83 M_{\odot}$, we calculated the minimum masses of the companions $m_{2} \sin i=$ $0.78 M_{\text {Jup }}$ for the inner and $m_{2} \sin i=2.93 M_{\text {Jup }}$ for the outer companion. The orbital semi-major axes are $0.07 \mathrm{AU}$ and $0.81 \mathrm{AU}$, respectively. The planetary orbits have moderate eccentricities of 0.35 and 0.27 , which seem to be not unusual, based on the statistics of the eccentricity of exoplanets (see e.g., http://www . exoplanet. eu).

When calculating the orbital solutions, we obtained a relatively large $\sigma(\mathrm{O}-\mathrm{C})$ value. A possible explanation to this is the presence of another unseen companion or RV jitter due to the interaction between the two companions. Because of the absence of the emission cores in $\mathrm{Ca}$ II $\mathrm{H} \& \mathrm{~K}$, the large $\sigma(\mathrm{O}-\mathrm{C})$ is most likely not due to the intrinsic stellar variability. 


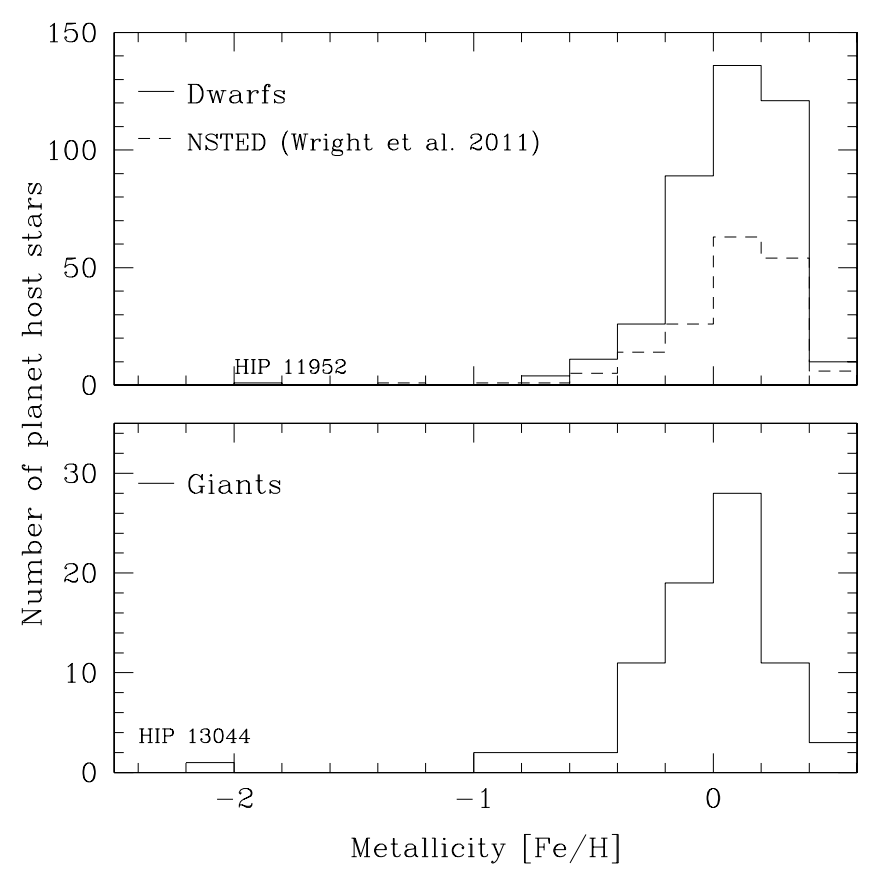

Fig. 10. The metallicity distribution of stars hosting planets. Solid lines in the top and bottom panels represent dwarfs and giants, respectively (data from the exoplanet encyclopedia http://www. exoplanet.eu). Dash lines show data from the exoplanet orbit database (Wright et al. 2011) which does not discriminate between dwarf and giant stars.

We investigated the residual velocities and found a significant signal at $\sim 40$ days. However, the amplitude of the residual $\mathrm{RV}$ variations is in the order of the error bars. Moreover, the window function shows also a peak close to this period. To detect further low-amplitude RV variations, more intensive highprecise RV measurements are needed.

From the knowledge of $P_{\text {rot }} / \sin i=15.7 \mathrm{~d}$ (Table 1) and the rotation period $P=4.82 \mathrm{~d}$, we derived an inclination angle of the stellar rotation of $18^{\circ}$. Assuming that the orbital inclination of the companion does not differ much from the stellar rotation inclination angle, we estimated true companion masses of 2.5 and $9.5 M_{\text {Jup }}$.

\section{Discussion}

A fundamental parameter of HIP 11952 is the stellar metallicity. The metallicity issue here is particularly interesting, since according to the theory of planet formation via core-accretion processes, planetary companions around such metal poor stars like HIP 11952 are not expected. The majority of the planet host main-sequence stars are metal rich (Fig. 10, upper panel).

For dwarf stars, the metallicity generally reflects the initial metallicity at star formation. Assuming that HIP 11952 is a dwarf or a turn off star, its initial low metallicity makes HIP 11952 unusual among the planetary systems discovered so far (Fig. 10).

The both planetary companions around HIP 11952 belong to only few planets that have been discovered in low metallicity systems $([\mathrm{Fe} / \mathrm{H}]<-1.5)$, comparable to HIP 13044 reported by Setiawan et al. (2010). Note that, so far also only few planets have been detected with host star's metallicities $-1<[\mathrm{Fe} / \mathrm{H}]<-0.5$. This group includes $\sim 60 \%$ main-sequence and $\sim 40 \%$ giants. From the current statistics, about $50 \%$ of the giant planet-host stars have sub-solar metallicities.

Whether the metallicity of a giant reflects its initial metallicity is still under debate. The convective envelopes in giant star are much more extended than in main-sequence stars (e.g. Pasquini et al. 2007) and they might alter their surface chemical abundances.

The presence and formation of planets around metal-poor stars, in particular those with metallicities $[\mathrm{Fe} / \mathrm{H}]<-1.5$ are still poorly understood. According to the core accretion theory (e.g., Safronov 1969; Pollack et al. 1996) high metallicity is required for the formation of planets. Alternatively, planets around metal-poor stars could form by gravitational disk instability processes (e.g., Boss 1998) or other mechanisms (see e.g., Nayakshin 2011). According to Nayakshin (2011) planets around such metal-poor stars have no solid cores and may form as a result of the second collapse (H2 disassociation) of their embryos and radial migrations. Further discoveries of planets around metal-poor stars can provide more constraints on currently different planet formation theories.

The planets around HIP 11952 have orbits with moderate eccentricities. To examine the dynamical stability of the system, numerical simulations are necessary to find stable planetary configurations, see e.g., Barnes \& Quinn (2004). Following their calculations, systems with two or more planets in large separated orbits are fully stable. The orbits of HIP $11952 \mathrm{~b}$ and $\mathrm{c}$ are in a 42:1 ratio and thus far beyond the 10:1 resonance. Therefore, the system HIP 11952 is most likely fully stable.

Finally, HIP 11952 and its planets are among the oldest planetary systems known. HIP 11952 is also older than typical stellar ages in the Galactic thick disk. A possible connection to a metal-poor stellar stream reported by Arifyanto \& Fuchs (2006) is an interesting aspect since it suggests that HIP 11952 might actually belong to a part of the thick disk that has been accreted from a disrupted former satellite galaxy, similar to HIP 13044. The age estimation of $12.8 \pm 2.6$ Gyr given by Feltzing et al. (2001) is 1 Gyr older than that of HD 114752 which has an estimated age of $11.8 \pm 3.9$ Gyr. HIP 11952's age is close to the one of HE 1523-0901, which is the oldest star (13.2 $\pm 2.7 \mathrm{Gyr})$ known so far (Frebel et al. 2007). The old stellar age is supported by the very low metallicity of HIP 11952 which is typical of Population II stars.

\section{Conclusions}

We observed RV variations of HIP 11952. The spectroscopic and photometric analysis of the star show that the periodic RV variations are not caused by the intrinsic stellar variability. Based on our analysis, we detected two planetary companions around the metal poor star HIP 11952 with orbital periods of $6.95 \mathrm{~d}$ and $290 \mathrm{~d}$. We found evidence for intra-day stellar pulsations and observed a stellar rotation of $4.82 \mathrm{~d}$. We computed the companion's minimum mass of $m_{2} \sin i=0.78 M_{\mathrm{Jup}}$ for the inner planet and $m_{2} \sin i=2.93 M_{\text {Jup }}$ for the outer one. Additional high-precise RV measurements are necessary to improve the orbital solution and put more constraints on the eccentricities. Further RV observations might also reveal the presence of other low-mass companions in the system. From the metal abundance analysis that we carried out, we obtained an average $[\mathrm{Fe} / \mathrm{H}]=-1.90 \pm 0.06$ from $\mathrm{Fe}_{\mathrm{I}}$ and $\mathrm{Fe}$ II, respectively, which makes HIP $11952 \mathrm{~b}$ and c the first planets discovered around a dwarf or subgiant star with $[\mathrm{Fe} / \mathrm{H}]<-1.5$. This discovery is also remarkable since the planetary system most likely belong to the first generation of planetary systems in the Milky Way. 
Acknowledgements. We thank N. Christlieb for the support and useful discussions. We also thank A. Mueller, M. Zechmeister, J. Carson, W. Wang, C. Ruhland, T. S. Hartung, S. Albrecht, R. Lachaume and T. Anguita for observing HIP 11952 with FEROS.

\section{References}

Arifyanto, M. I., \& Fuchs, B. 2006, A\&A, 449, 533

Barnes, R., \& Quinn, Th. 2004, ApJ, 611, 494

Bessell, M. S. 2000, PASP, 112, 961

Bouchy, F., Hebb, L., Skillen, I., et al. 2010, A\&A, 519, A98

Boss, A. P. 1998, ApJ, 503, 923

Caffau, E., Ludwig, H.-G., Steffen, M., Freytag, B., \& Bonifacio, P. 2010, Sol. Phys., 66

Caffau, E., Ludwig, H.-G., Steffen, M., Freytag, B., \& Bonifacio, P. 2011, Sol. Phys., 268, 255

Casagrande, L., Ramírez, I., Meléndez, J., Bessell, M., \& Asplund, M. 2010, A\&A, 512, A54

Cayrel de Strobel, G., Soubiran, C., Friel, E. D., et al. 1997, A\&A, 124, 299

Cayrel de Strobel, G., Soubiran, C., \& Ralite, N. 2001, A\&A, 373, 159

Carney, B., \& Latham, D. 1987, AJ, 93, 116

Cenarro, A. J., Peletier, R. F., Sánchez-Blázquez, P., et al. 2007, MNRAS, 374, 664

Charbonnel, C., \& Primas, F. 2005, A\&A, 442, 961

de Medeiros, J. R., \& Mayor, M. 1999, A\&AS, 139, 433

Eggen, O. J., \& Sandage, A. R. 1959, MNRAS, 119, 225

Edvardsson, B., Andersen, J., Gustafsson, B., et al. 1993, A\&A, 275, 101

Feltzing, S., Holmberg, J., \& Hurley, J. R. 2001, A\&A, 377, 911

Fischer, D. A., \& Valenti, J. 2005, ApJ, 622, 1102

Fouts, G. 1987, PASP, 99, 986

Frebel, A., Christlieb, N., Norris, J. E., et al. 2007, ApJ, 660, L117

Fulbright, J. P. 2002, AJ, 123, 404

Girardi, L., Williams, B. F., Gilbert, K. M., et al. 2010, ApJ, 724, 1030

Gratton, R. G., Carretta, E., \& Castelli, F. 1996, A\&A, 314, 191

Gray, R. O., \& Corbally, C., J. 2009, Stellar Spectral Classification, ed. R. O. Gray, \& C. J. Corbally (Princeton University Press)

Hatzes, A. P. 1996, PASP, 108, 839

Johnson, J. A., Aller, K. M., Howard, A. W., \& Crepp, J. R. 2010, PASP, 122, 905

Kaufer, A., \& Pasquini, L. 1998, Proc. SPIE, 3355, 844
Kharchenko, N. V., \& Roeser, S. 2009, VizieR Online Data Catalog, 1280

Klement, R. J. 2010, A\&ARv, 18, 567

Kurucz, R. 1993, ATLAS9 Stellar Atmosphere Programs and $2 \mathrm{~km} \mathrm{~s}^{-1}$ grid Kurucz CD-ROM No. 13 (Cambridge, Mass.: Smithsonian Astrophysical Observatory)

Kurucz, R. L. 2005, Mem. Soc. Astron. Ital. Suppl., 8, 14

Larson, A. M., Irwin, A. W., Yang, S. L. S., et al. 1993, PASP, 105, 332

Marigo, P., Girardi, L., Bressan, A., et al. 2008, A\&A, 482, 883

Masana, E., Jordi, C., \& Ribas, I. 2006, A\&A, 450,735

Minchev, I., Quillen, A. C., Williams, M., et al. 2009, MNRAS, 396, L56 Nayakshin, S. 2011, MNRAS, 416, 2974

Pasquini, L., Döllinger, M. P., Weiss, et al. 2007, A\&A, 473, 979

Perryman, M. A. C., \& ESA 1997, ESA SP Ser., 1200

Pollack, J. B., Hubickyj, O., Bodenheimer, P., et al. 1996, Icarus, 124, 62

Roberts, D. H., \& Dreher, J.W. 1987, AJ, 93, 968

Rodríguez-Ledesma, M. V., Mundt, R., \& Eislöffel, J. 2009, A\&A, 502, 883

Safronov, V. S. 1969, Evoliutsiia do planetnogo oblaka

Sánchez-Blázquez, P., Peletier, R. F., \& Jiménez-Vicente, J. 2006, MNRAS, 371, 703

Santos, N. C., Mayor, M., Naef, D., et al. 2000, A\&A, 361, 265

Santos, N. C., Mayor, M., Benz, W., et al. 2010, A\&A, 512, A47

Santos, N. C., Mayor, M., Bonfils, X., et al. 2011, A\&A, 526, A112

Sbordone, L. 2005, Mem. Soc. Astron. Ital. Suppl., 8, 61

Sbordone, L., Bonifacio, P., Castelli, F., \& Kurucz, R. L. 2004, Mem. Soc. Astron. Ital. Suppl., 5, 93

Scargle, J, D. 1982, ApJ, 263, 835

Scholz, A., \& Eislöffel, J. 2004, A\&A, 421, 259

Setiawan, J., Pasquini, L., da Silva, L., von der Lühe, O., \& Hatzes, A. 2003, A\&A, 397, 1151

Setiawan, J., Klement, R. J., Henning, T., et al. 2010, Science, 330, 1642

Simpson, E. K., Barros, S. C. C., Brown, D. J. A., et al. 2011, AJ, 141, 8

Sivarani, T., Bonifacio, P., Molaro, P., et al. 2004, A\&A, 413, 1073

Sozzetti, A., Torres, G., Latham, D. W., et al. 2009, ApJ, 697, 544

Valdes, F., Gupta, R., Rose, J. A., Singh, H. P., \& Bell, D. J. 2004, ApJS, 152, 251

Valenti, J. A., \& Fischer, D. A. 2005, ApJS, 159, 141

Vaughan, A. H., Preston, G., W., \& Wilson, O. C. 1978 PASP, 90, 267

Wright, C. O., Egan, M. P., Kraemer, K. E., \& Price, S. D. 2003, AJ, 125, 359

Wright, J. T., Fakhouri, O., Marcy, G. W., et al. 2011, PASP, 123, 412

Zechmeister, M., \& Kürster, M. 2009, A\&A, 496, 577

Zerbi, F. M. 2000, in Delta Scuti and Related Stars, ASP Conf. Ser., 210, 332 